\title{
Integrated analysis of transcriptome and metabolites reveals an essential role of metabolic flux in starch accumulation under nitrogen starvation in duckweed
}

Changjiang Yu ${ }^{1,2} \oplus$, Xiaowen Zhao ${ }^{3}$, Guang Qi ${ }^{1}$, Zetao Bai ${ }^{1}$, Yu Wang ${ }^{1}$, Shumin Wang ${ }^{1}$, Yubin Ma ${ }^{1}$, Qian Liư , Ruibo $\mathrm{Hu}^{1 *}$ and Gongke Zhou ${ }^{1^{*}}$

\begin{abstract}
Background: Duckweed is considered a promising source of energy due to its high starch content and rapid growth rate. Starch accumulation in duckweed involves complex processes that depend on the balanced expression of genes controlled by various environmental and endogenous factors. Previous studies showed that nitrogen starvation induces a global stress response and results in the accumulation of starch in duckweed. However, relatively little is known about the mechanisms underlying the regulation of starch accumulation under conditions of nitrogen starvation.

Results: In this study, we used next-generation sequencing technology to examine the transcriptome responses of Lemna aequinoctialis 6000 at three stages $(0,3$, and 7 days) during nitrogen starvation in the presence of exogenously applied sucrose. Overall, 2522, 628, and 1832 differentially expressed unigenes (DEGs) were discovered for the treated and control samples. Clustering and enrichment analysis of DEGs revealed several biological processes occurring under nitrogen starvation. Genes involved in nitrogen metabolism showed the earliest responses to nitrogen starvation, whereas genes involved in carbohydrate biosynthesis were responded subsequently. The expression of genes encoding nitrate reductase, glutamine synthetase, and glutamate synthase was down-regulated under nitrogen starvation. The expression of unigenes encoding enzymes involved in gluconeogenesis was up-regulated, while the majority of unigenes involved in glycolysis were down-regulated. The metabolite results showed that more ADP-Glc was accumulated and lower levels of UDP-Glc were accumulated under nitrogen starvation, the activity of AGPase was significantly increased while the activity of UGPase was dramatically decreased. These changes in metabolite levels under nitrogen starvation are roughly consistent with the gene expression changes in the transcriptome.

Conclusions: Based on these results, it can be concluded that the increase of ADP-glucose and starch contents under nitrogen starvation is a consequence of increased output from the gluconeogenesis and TCA pathways, accompanied with the reduction of lipids and pectin biosynthesis. The results provide novel insights into the underlying mechanisms of starch accumulation during nitrogen starvation, which provide a foundation for the improvement of advanced bioethanol production in duckweed.
\end{abstract}

Keywords: Duckweed, Transcriptome sequencing, Nitrogen stress, Metabolic flux, Starch accumulation

\footnotetext{
*Correspondence: hurb@qibebt.ac.cn; zhougk@qibebt.ac.cn

${ }^{1}$ Shandong Provincial Key Laboratory of Energy Genetics, Qingdao Engineering Research Center of Biomass Resources and Environment, Qingdao Institute of Bioenergy and Bioprocess Technology, Chinese Academy of Sciences, Qingdao 266101, People's Republic of China Full list of author information is available at the end of the article
} 


\section{Background}

With increasing dependence on fossil fuels, which is unsustainable and has increasingly high environmental, economic, and political costs, there has been a push to identify alternative renewable energy sources $[1,2]$. Ethanol production from dedicated bioenergy crops or agricultural residues is one of the more promising technologies that can help address this pressing issue. Corn is currently the predominant feedstock for ethanol production in China. However, corn-based ethanol production endangers domestic food security because it competes with food and feed production due to limited agricultural land [3]. Therefore, there is an urgent need to explore alternative feedstock sources for ethanol production [4].

Species of the Lemnaceae family (duckweed) are the world's smallest, but fastest growing, aquatic flowering plants. Lemnaceae is a monocotyledonous family with 37 species in five genera: Spirodela, Landoltia, Lemna, Wolffia, and Wolffiella [5]. Duckweed is commonly used for bioremediation of nutrient-rich wastewater and as fodder for fish and ducks due to its ability to absorb substantial amounts of nutrients and high protein content. However, recent research has demonstrated that duckweed can also be regarded as a potential feedstock for bioethanol production due to its impressive ability for starch accumulation [6-8]. The starch content of duckweed varies significantly (dry weight) depending on the species and growing conditions $[4,9]$. The effects of various stress conditions, such as nitrogen starvation, temperature extremes, and salt, on starch accumulation in duckweed have been addressed $[8,10]$. However, compared to a model plant (i.e., Arabidopsis thaliana), the molecular mechanism of starch accumulation during physiological stress remains largely unknown in other plant species, especially in duckweed. Although the genome sequences of duckweed species (Spirodela polyrhiza and Lemna minor) have been released, the availability of the genomic sequences is somewhat restricted because the genome sizes vary enormously, ranging from $150 \mathrm{Mb}$ in Spirodela to $1881 \mathrm{Mb}$ in Wolffia, which is a 13-fold difference [11]. However, with the rapid evolution of next-generation sequencing strategies and decreases in sequencing costs, transcriptome sequencing serves as an alternative and more efficient approach because it targets only coding DNA, which can facilitate the de novo assembly of transcriptomes from non-model species $[12,13]$.

To date, three studies of transcriptome analysis in duckweed species have been published. A study in Landoltia punctate showed that the gene expression level of starch degradation genes were negatively correlated with biosynthesis genes, which led to starch accumulation [14]. Another RNA-Seq study focused on the transcriptome analysis of Spirodela dormancy without reproduction and showed that key genes involved in starch synthesis (e.g., $A P S 1, A P L 3$, and GBSSI) were triggered by the application of exogenous ABA [15]. A third RNA-seq analysis revealed dose rate-dependent gene expression responses and indicated that Lemna minor shifts from acclimation responses towards survival responses at increasing dose rates of ionizing radiation [16]. Currently, the molecular mechanism of a single stressor on starch accumulation in duckweed is yet to be revealed. In the current study, we performed a transcriptome survey to reveal the molecular mechanism of starch accumulation under nitrogen starvation in L. aequinoctialis 6000, which is a fast-growing and high starch species of duckweed.

In this study, we analyzed the transcriptomic profiles and metabolite patterns of duckweed (Lemna aequinoctialis 6000) during a 7-day time course under nitrogen starvation. Differentially expressed genes (DEGs) were identified and categorized as various functional classifications and nitrogen starvation-related metabolic metabolisms, which were further validated by examining specific enzyme activities. The results demonstrate the feasibility of using transcriptome data with a next-generation sequencing (NGS) method to identify interesting pathways and potential target genes, which could serve as excellent candidates for functional genomics studies and metabolic engineering to improve the production of next-generation biofuels in duckweed.

\section{Methods}

\section{Plant materials and growth conditions}

Lemna aequinoctialis 6000 was obtained from a screening of more than 100 duckweed species distributed across 20 provinces in China [17]. The strain used in this study was collected from Li Xian in Hunan Province. The plants were cultured in a growth chamber at $24{ }^{\circ} \mathrm{C}$ under a $16 \mathrm{~h}$ light $/ 8 \mathrm{~h}$ dark photoperiod and at a light intensity of $110 \mu \mathrm{mol} \mathrm{m} \mathrm{m}^{-2} \mathrm{~s}^{-1}$ provided by wide spectrum fluorescent tubes. The light intensity was measured using a quantum photometer (LI-250A, LI-COR, USA). The plants were grown in liquid Schenk and Hildebrandt (SH) medium supplemented with $10 \mathrm{~g} / \mathrm{L}$ sucrose for 2 weeks, then transferred to liquid $\mathrm{SH}$ medium that lacked nitrogen but was also supplemented with $10 \mathrm{~g} / \mathrm{L}$ sucrose. Samples were collected at three different time points $(0,3$, and 7 days) at 2 pm for the RNA-Seq analysis. The sampling time points were determined based on a pilot experiment that examined the expression of the ADPglucose pyrophosphorylase gene, which is an important marker gene in starch biosynthesis [18].

\section{Starch content and periodic acid-Schiff (PAS) staining}

Starch granules were stained using the Periodic AcidSchiff (PAS) Kit (Sigma-Aldrich) according to the 
manufacturer's instructions. Total starch content was determined using a Megazyme total starch assay kit. Briefly, $100 \mathrm{mg}$ of frozen dry duckweed was ground into powder with a multi-tube ball mill (Tissuelyser II, Qiagen, Germany) and transferred into a test tube. To aid dispersion, $0.2 \mathrm{~mL}$ of $80 \%$ ethanol was stirred into the sample with a vortex mixer. The tube was then placed in a $50^{\circ}$ water bath, and $4 \mathrm{~mL}$ of sodium acetate was added, followed by the addition of $\sim 3$ units of thermostable alpha-amylase (Sigma A4582, USA) and $~ 6$ units amyloglucosidase (Sigma A7095, USA). The sample tube was then agitated with a vortex mixer and incubated for $30 \mathrm{~min}$ in a $50{ }^{\circ} \mathrm{C}$ water bath. The contents were then moved/washed into a $100 \mathrm{~mL}$ volumetric flask with distilled water, mixed, and centrifuged at $3000 \mathrm{rpm}$ for $10 \mathrm{~min}$. A $0.1 \mathrm{~mL}$ aliquot of the supernatant was added to $2 \mathrm{~mL}$ of GOPOD reagent, vortexed, and incubated at $50{ }^{\circ} \mathrm{C}$ for $20 \mathrm{~min}$. Blank $(0.1 \mathrm{~mL}$ of distilled water and $2 \mathrm{~mL}$ of GOPOD) and glucose standards (0.2, 0.4, 0.6, 0.8, and $1.0 \mathrm{mg} / \mathrm{mL}$ ) were incubated concurrently. Absorbance was measured with a spectrophotometer at $510 \mathrm{~nm}$ with a path length of $1 \mathrm{~cm}$.

\section{RNA extraction and library construction}

Total RNA was extracted with Trizol reagent (Invitrogen, USA) and then treated with RNase-Free DNase I (TaKaRa, Japan) to remove DNA contaminants. RNA integrity was confirmed using agarose gel electrophoresis and an Agilent 2100 Bioanalyzer (Agilent, USA). RNA concentrations were determined using a Qubit 2.0 spectrophotometer (Life Technology, USA). For each treatment, mRNA was purified from $10 \mu \mathrm{g}$ of total RNA using magnetic oligo (dT) beads and fragmented using fragmentation buffer with the preparation kit according to the manufacturer's instructions (Invitrogen, USA). The cleaved short RNA fragments were used for first-strand cDNA synthesis using reverse transcriptase and hexamer primer (Illumina), and the second strand cDNA was synthesized with DNA polymerase I and RNase $\mathrm{H}$ (Thermo Fisher, USA). cDNA fragments were selected for PCR amplification, and cDNA libraries were used for sequencing with the Illumina HiSeq ${ }^{\mathrm{TM}} 2000$ platform.

\section{Data filtering and de novo assembly}

Raw reads obtained after sequencing were firstly filtered by removing reads containing adapters, reads containing more than $10 \%$ of unknown nucleotides $(\mathrm{N})$, and low quality reads containing more than $50 \%$ of low quality $(Q<20)$ bases using custom Perl scripts. The clean reads were then assembled into unique consensus sequences using Trinity [19]. The redundancy was eliminated using TGICL [20] and further assembled into a single set of non-redundant unigenes.

\section{Functional annotation of unigenes}

Six databases were used for unigene annotation, including the NCBI protein database $(\mathrm{Nr})$, the NCBI nucleotide database (Nt), the euKaryotic Orthologous Groups database (KOG), the Swiss-Prot database, the KEGG Orthology database, and the Gene Ontology (GO) databases. These annotations were performed using a combination of BLAST, Blast2GO, HMMER, and KEGG Automatic Annotation Server (KAAS) tools.

\section{Analysis of differential expression genes}

Using the transcriptome assembled by Trinity [19] as a reference, clean reads of each sample were mapped by RSEM [21]. The normalized read counts from two replicates of each sample were analyzed, and unigenes showing significant differences in expression were determined using DEseq [22]. The FDR $q$ value threshold was set to 0.005 , and the fold change of expression was set to 2.0 .

\section{Gene ontology and Kyoto Encyclopedia of Genes and Genomes (KEGG) enrichment analysis}

Gene ontology and KEGG enrichment analysis was carried out to determine the distributions of unigenes based on their functions and biological pathways. GO analysis was performed using Goseq based on Wallenius' noncentral hypergeometric distributions [23]. KEGG enrichment analysis was performed using KOBAS 2.0 with hypergeometric tests [24]. GO categories and KEGG pathways with FDR $q$ value $\leq 0.05$ were considered to be significantly enriched.

\section{Quantitative real-time RT-PCR}

Quantitative real-time RT-PCR (qRT-PCR) was performed to validate the expression of unigenes. Genespecific primers for seven randomly selected DEGs were designed by Beacon Designer 8.0. The $18 \mathrm{~S}$ gene was used as the internal control. First-strand cDNA was synthesized using TransScript One-Step gDNA Removal and cDNA Synthesis SuperMix (TransGen Biotech, China) according to the manufacturer's instructions. Reactions were carried out using a SYBR Premix Ex Taq II (Tli RnaseH Plus) Kit (TaKaRa, Japan) in a Lightcycler 480 Real-Time PCR Detection System (Roche, Germany). Amplification reaction mixtures consisted of $10 \mu \mathrm{L}$ of SYBR Premix Ex Taq II, $0.2 \mu \mathrm{L}$ of each forward and reverse primer $(10 \mathrm{mM})$, and $1 \mu \mathrm{L}$ of cDNA template, and sterile water was added to a final volume of $20 \mu \mathrm{L}$. The amplification cycling program was as follows: $94{ }^{\circ} \mathrm{C}$ for $30 \mathrm{~s}, 42$ cycles of $94{ }^{\circ} \mathrm{C}$ for $5 \mathrm{~s}$, and $59^{\circ} \mathrm{C}$ for $30 \mathrm{~s}$. The relative expression was calculated using the $2^{-\Delta \Delta C T}$ method [25]. qRT-PCR analysis was conducted with three technical replicates, and the data represent the means \pm standard errors $(n=3)$. 
Carbohydrate measurement and enzyme activity assays

Leaves were flash frozen, then extracted in chloroform/ methanol. Starch, soluble glucans, and sugars were extracted and assayed by enzymatic methods [26]. To examine the enzyme activities, $1 \mathrm{~g}$ of fresh duckweed was homogenized with a ceramic pestle in an ice-cold mortar in $5 \mathrm{~mL}$ of reaction solution [50 mM HEPES-NaOH (pH 7.6), $5 \mathrm{mM}$ DL-dithiothreitol, $8 \mathrm{mM} \mathrm{MgCl}_{2}, 2 \mathrm{mM}$ EDTA, 2\% (w/v) PVP-40, and 12.5\% (w/v) glycerol]. The homogenate was centrifuged at $10,000 \times g$ for $5 \mathrm{~min}$. The supernatant was used as a crude enzyme solution and stored at $-20{ }^{\circ} \mathrm{C}$. The activities of $\alpha$-amylase $(1,4, \mathrm{D}$-glucan glucanohydrolase, 3.2 .1 .1$)$ and $\beta$-amylase $(1,4$, D-glucan maltohydrolase, 3.1.3.12) were determined following the method of Tarrago and Nicolas [27]. The enzymatic activities of soluble starch synthases (SSs, 2.4.1.21) and AGPase (2.7.7.9) were assayed according to Nakamura et al. [28]. Starch synthase activity was assayed according to Jenner et al. [29]. To visualize glycogen and starch synthase activities, leaves were extracted with $100 \mathrm{mM}$ MOPS (pH 7.2), 1 mM EDTA, 1 mM DTT, and 10\% (w/v) glycerol (300 mg sample/mL). Extracts were loaded onto non-denaturing polyacrylamide gels containing $0.3 \%$ $(\mathrm{w} / \mathrm{v})$ glycogen. After incubation for $16 \mathrm{~h}$ at $20{ }^{\circ} \mathrm{C}$ in $100 \mathrm{mM}$ HEPES-NaOH (pH 7.5), 2 mM DTT, 10\% (v/v) glycerol, $0.5 \mathrm{mM}$ EDTA, $0.5 \mathrm{M}$ sodium citrate, and $2 \mathrm{mM}$ ADP-glucose, both starch and glycogen synthase activities were revealed by iodine staining.

\section{Results}

Impact of nitrogen starvation in the presence of exogenously applied sucrose on starch accumulation

The starch content of L. aequinoctialis 6000 grown under nitrogen starvation conditions was measured at three different time points. The initial starch content was measured at approximately $20 \%$ before treatment and then increased rapidly over the following 9 days during nitrogen starvation (Fig. 1A). The highest starch content was $60 \pm 3 \%$ after treatment for 9 days, which was almost three times higher compared to the pre-treatment. The results showed that $L$. aequinoctialis had considerable starch accumulation during nitrogen starvation. Therefore, this plant can be used as an ideal experimental model to characterize the mechanisms underlying starch accumulation. We further determined the starch granule content through in situ staining of fronds with periodic acid-Schiff (PAS) (Fig. 1B). The results indicated that the number of starch granules significantly increased under nitrogen starvation, which was consistent with the results in Fig. 1A. The starch granules were sparsely distributed in fronds at the cell edge (Fig. 1B (a)) and then gradually accumulated to the cell center (Fig. $1 \mathrm{~B}(\mathrm{~b}-\mathrm{d})$ ) as the starch content increased. The starch granules occupied almost the entire cell after 7 days of nitrogen starvation (Fig. 1B (d)).

\section{Illumina HiSeq mRNA sequencing}

To identify the genes responsible for starch accumulation, we explored the transcriptome profile of $L$. aequinoctialis 6000 during nitrogen starvation using RNA-seq. Overall, $25 \mathrm{G}$ sequencing data were generated and de novo assembled into 72,105 unigenes with an average length of $1233 \mathrm{bp}$ (Table 1; Additional file 1: Table S1; Additional file 2: Table S2).

Functional annotation of the unigenes was carried out in a BLAST search of public databases, including the NCBI Nr and Nt database, the GO database, the KOG database, the Swiss-Prot protein database, and the KEGG database. Overall, 53,734 (74.52\%) unigenes were functionally annotated according to their similarities with known genes/proteins in these databases. The detailed statistics of the functional annotation are provided in Additional file 3: Figure S1.

\section{Identification of differentially expressed genes during nitrogen starvation}

The expression profiles of L. aequinoctialis cultivated under nitrogen starvation conditions were analyzed. Compared to the samples at 0 day, the expression of 2522 unigenes changed after nitrogen starvation for 3 days, whereas the expression of 628 unigenes were affected at 7 days under nitrogen starvation. There were 323 differentially expressed genes (DEGs) in both the 3- and 7-day treatments (Fig. 2a). All DEGs were subjected to GO term analysis (Additional file 4: Figure S2), KOG analysis (Additional file 5: Figure S3), and KEGG pathway enrichment analysis (Additional file 6: Figure S4). There were 157,910 (48.66\%) unigenes assigned to a biological process, 123,574 (38.08\%) unigenes assigned to a cellular component, and 43,007 (13.25\%) unigenes assigned to a molecular function. Overall, 35,529 unigenes (49.27\%) were classified into 25 KOG categories. Among the $25 \mathrm{KOG}$ categories, the "cluster of general function prediction" (9560, 26.91\%) represented the largest group, followed by "transcription" (5851, $16.47 \%)$, "replication, recombination, and repair" (5089, 14.32\%), "signal transduction mechanisms" (4359, $12.26 \%)$, "translation, ribosomal structure, and biogenesis" (3521, 9.91\%), and "carbohydrate transport and metabolism" (3461, 9.74\%). A total of 35,529 (49.27\%) unigenes had hits for 128 KEGG pathways. "Metabolic pathways" contained the most unigenes (8546, 24.05\%), followed by "Biosynthesis of secondary metabolites" (3719, 10.47\%), "RNA transport" (2330, 6.56\%), and "Spliceosome" (2119, 5.96\%). The remaining 117 KEGG pathways each contained less than 1000 unigenes. 
A

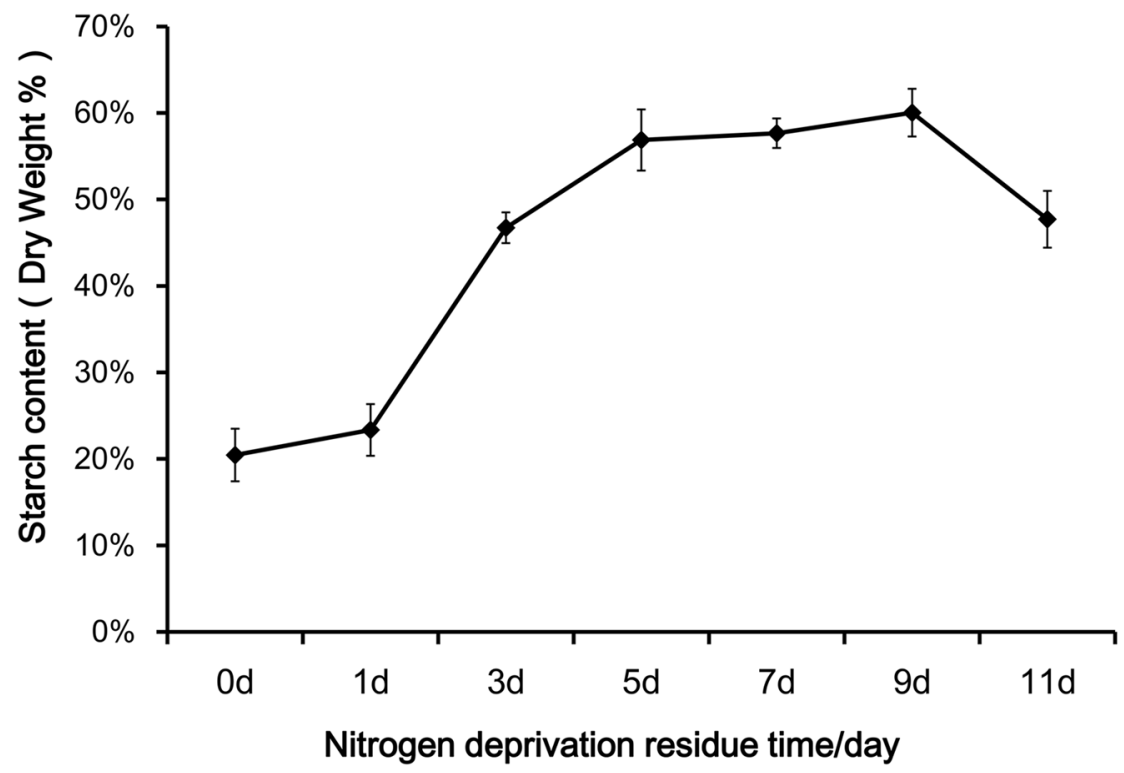

B
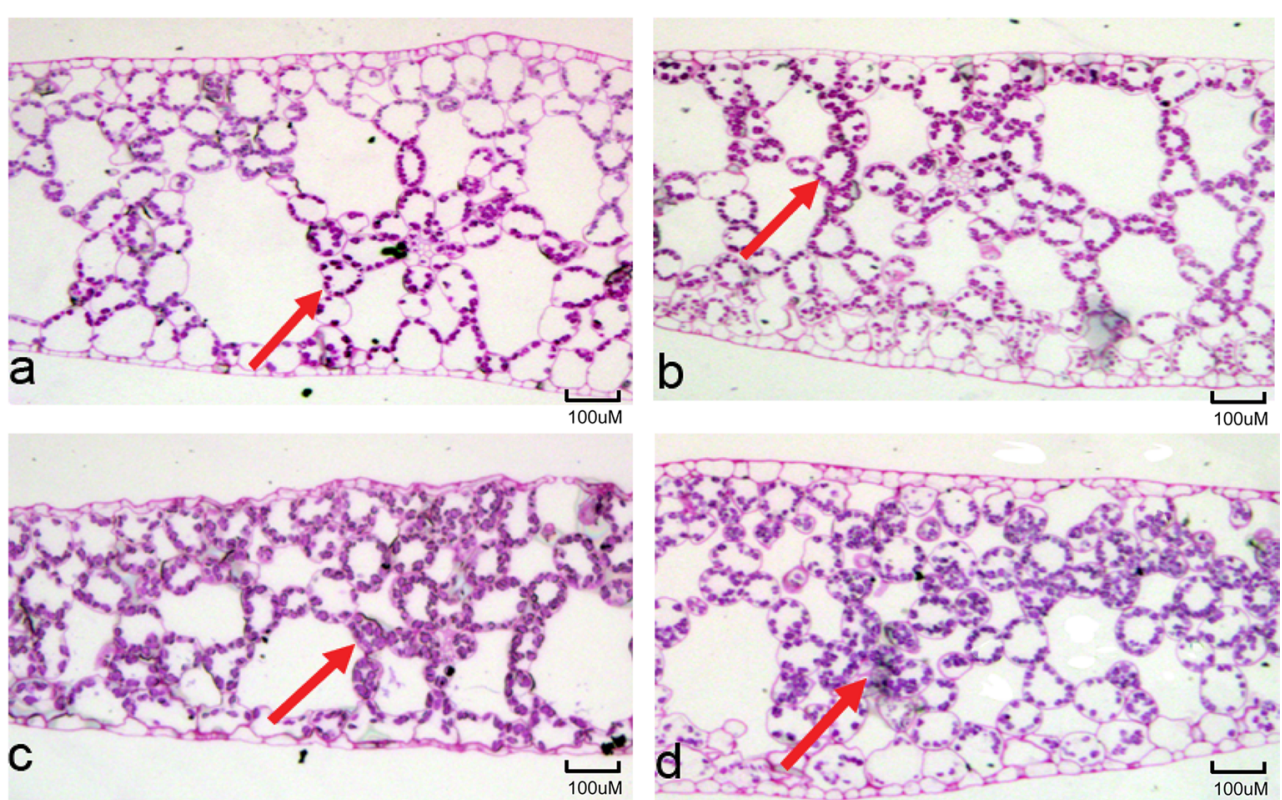

Fig. 1 Starch accumulation in L. aequinoctialis 6000 during nitrogen starvation. A Quantification of starch contents at different stages of nitrogen starvation. B Cross sections of duckweed fronds showing accumulation of starch granules during nitrogen starvation. a-d indicate 0 day (a), 3 days (b), 5 days (c), and 7 days (d) during nitrogen starvation. Red arrows indicate the starch granules stained with periodic acid-Schiff (PAS)

To further explore the biological function of these DEGs, hierarchical clustering was performed using the Euclidean distance associated with complete-linkage analysis (Fig. 2b). Expression pattern analysis was also carried out, and a total of eight clusters were identified (cluster 0-cluster 7, Fig. 3). The enrichment analysis was performed for each profile to identify the putative pathways associated with starch accumulation under nitrogen starvation (Additional file 7: Figure S5, Additional file 8: Figure S6).

As shown in Fig. 3, the genes in cluster 2 and cluster 5 represented two of the largest of DEGs with 2476 and 625 genes, respectively, and the genes in these two clusters had opposite expression patterns. The genes in cluster 2 were significantly down-regulated at 3 days compared to the 0 and 7 days levels during nitrogen starvation. In 
Table 1 Assembly quality statistics of the L. aequinoctialis transcriptome

\begin{tabular}{|c|c|c|c|c|c|c|c|c|}
\hline Samples & Total reads & $\begin{array}{l}\text { Total unigene } \\
\text { number }\end{array}$ & $\begin{array}{l}\text { Total length } \\
\text { (nt) }\end{array}$ & $\begin{array}{l}\text { Mean length } \\
\text { (nt) }\end{array}$ & N50 & $\begin{array}{l}\text { Total consensus } \\
\text { sequence }\end{array}$ & $\begin{array}{l}\text { Distinct } \\
\text { clusters }\end{array}$ & $\begin{array}{l}\text { Distinct } \\
\text { singletons }\end{array}$ \\
\hline $0 d-1$ & $45,631,688$ & 64,360 & $56,557,875$ & 879 & 1687 & 64,360 & 35,175 & 29,185 \\
\hline $0 d-2$ & $42,041,558$ & 51,699 & $40,541,311$ & 784 & 1453 & 51,699 & 28,052 & 23,647 \\
\hline $3 d-1$ & $33,961,764$ & 51,664 & $42,605,247$ & 825 & 1559 & 51,664 & 27,845 & 23,819 \\
\hline $3 d-2$ & $33,586,294$ & 51,250 & $40,967,920$ & 799 & 1523 & 51,250 & 27,545 & 23,705 \\
\hline $7 d-1$ & $46,889,100$ & 58,605 & $52,433,518$ & 895 & 1681 & 58,605 & 33,003 & 25,602 \\
\hline $7 d-2$ & $44,459,216$ & 50,940 & $41,122,923$ & 807 & 1539 & 50,940 & 27,090 & 23,850 \\
\hline All & & 72,105 & $88,895,700$ & 1233 & 1916 & 72,105 & 47,937 & 24,168 \\
\hline
\end{tabular}

a

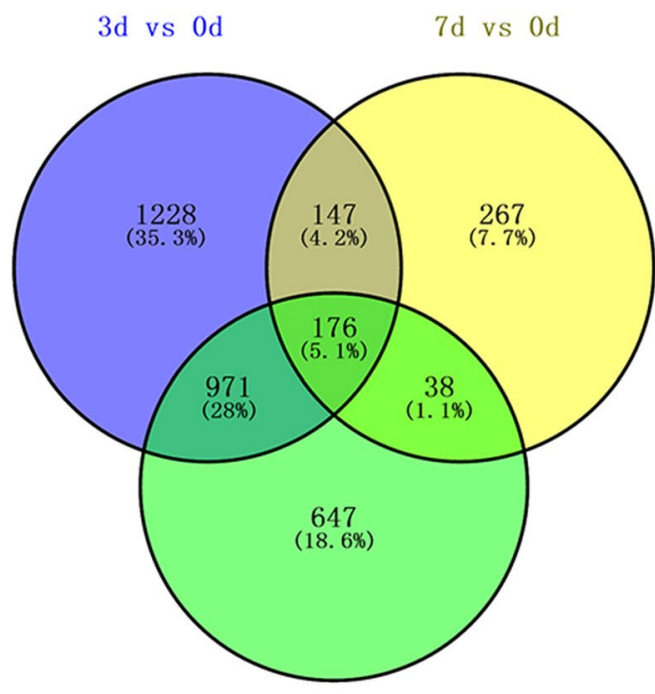

$7 \mathrm{~d}$ vs $3 d$ b

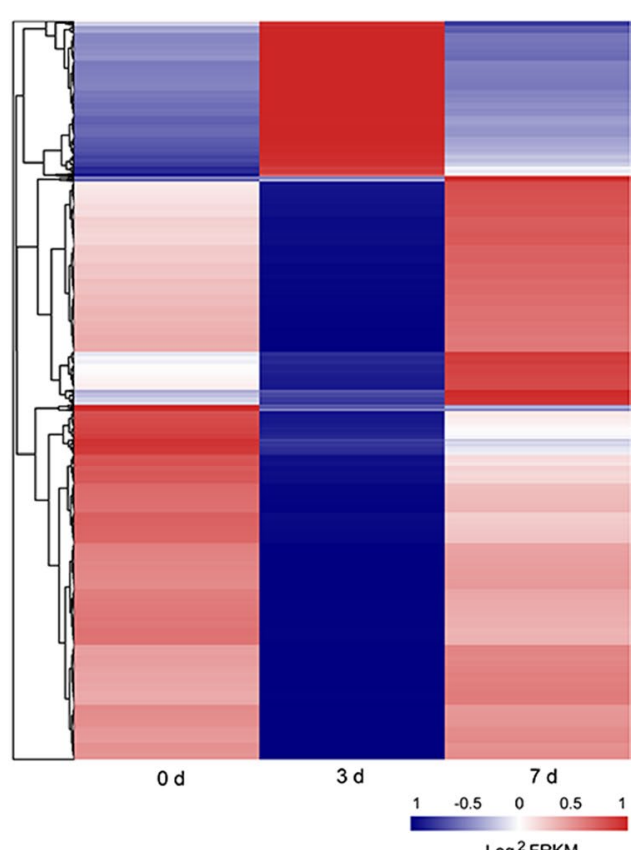

Fig. 2 Statistics of the differentially expressed genes in L. aequinoctialis during nitrogen starvation. a Venn diagram showing differentially expressed genes at 3 and 7 days after nitrogen starvation compared to the 0 day control; $\mathbf{b}$ hierarchical clustering of all differentially expressed genes (DEGs) based on normalized FPKM values in three time points. Blue indicates lower expression, and red indicates higher expression

contrast, the genes in cluster 5 were significantly up-regulated at 3 days compared to the other time points during nitrogen starvation. Because cluster 2 and cluster 5 represented two of the largest groups of DEGs, we conducted an enrichment analysis of functional categories for DEGs in these two clusters. The significantly enriched categories in cluster 2 included nitrogen metabolism-related pathways as well as pathways involved in the light reaction of photosynthesis, carotenoid biosynthesis, amino acid biosynthesis, carbon metabolism, and unsaturated fatty acid biosynthesis. The enriched categories in cluster 5 included starch and sugar metabolism, glycan degradation, glyoxylate and dicarboxylate metabolism, and riboflavin metabolism.

\section{Pathways induced by nitrogen starvation}

Seventy-two DEGs were enriched in starch and sucrose metabolism pathways, and more than 100 DEGs related to the starch pathways were differentially expressed during nitrogen starvation. Starch biosynthesis involves a series of key enzymes including soluble starch synthase (SS), ADP-glucose pyrophosphorylase (AGP), granule bound starch synthase (GBSS), and starch-branching enzyme (SBE), whereas the degradation of starch is catalyzed by $\alpha$-amylase and $\beta$-amylase [30]. Transcripts encoding AGP and SS were significantly up-regulated under nitrogen starvation (Figs. 4, 5). However, the expression of transcripts encoding GBSS and SBE under nitrogen starvation showed no significant differences 


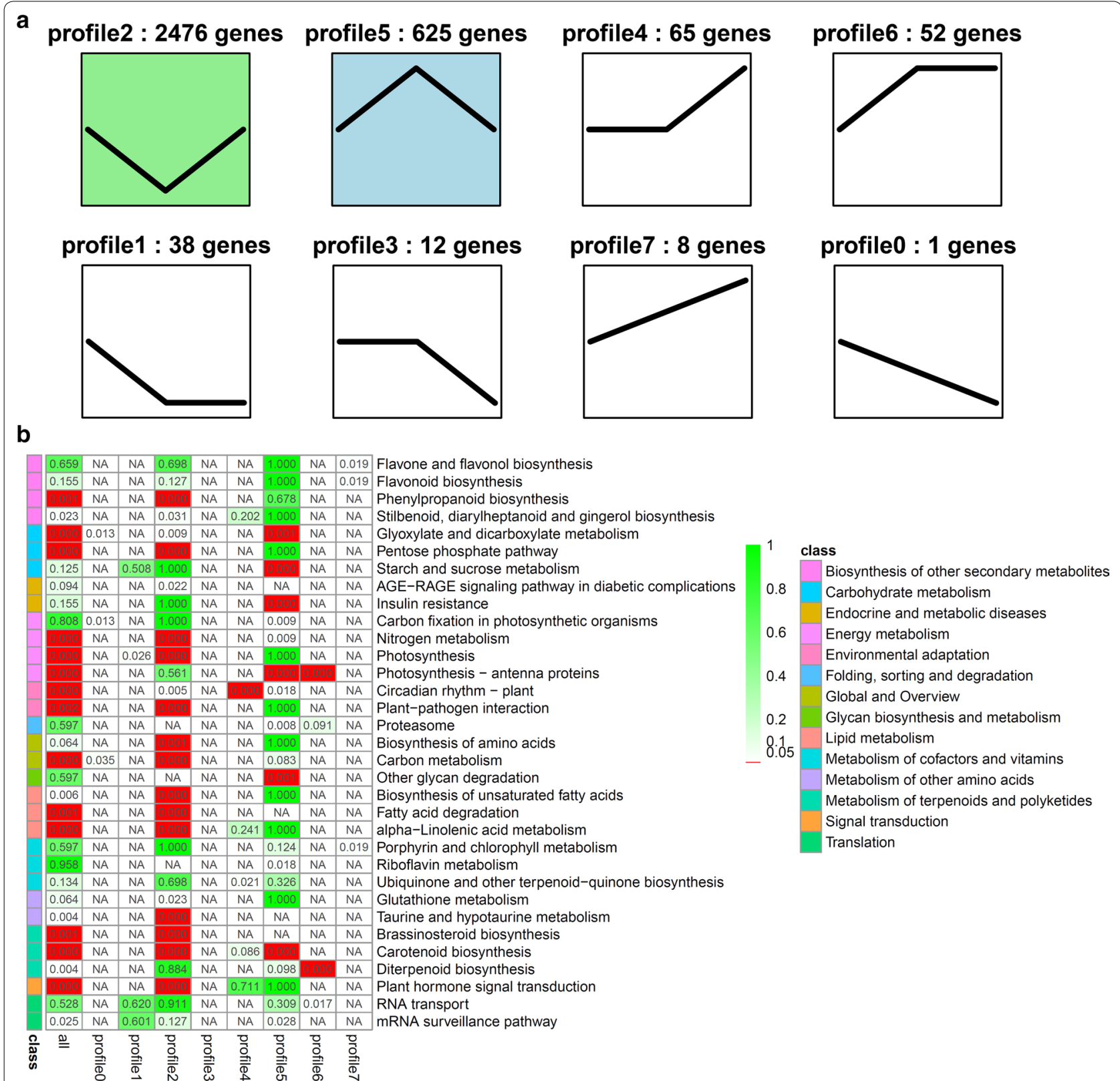

Fig. 3 Dynamic transcriptome of L. aequinoctialis during nitrogen starvation. a Cluster of gene expression patterns in response to nitrogen starvation. Short time-series expression miner (STEM) was used to analyze the gene expression pattern, and eight profiles exhibited significant clustering of gene expression patterns. The number of unigenes in each profile was labeled above the frame. The black line represents the general tendency in each profile. Expression patterns of 8 profiles at different stages under nitrogen starvation. $\mathbf{b}$ Enrichment of functional categories of each cluster with the significantly enriched KEGG pathways plotted for DEGs among the three stages of nitrogen starvation

from the untreated samples. In addition, the expression of genes encoding enzymes related to glycolysis, gluconeogenesis, UDP-glucose, GDP-glucose, and $\alpha$-Dglucose-1P were also analyzed under nitrogen starvation (Figs. 4, 5). The expression of unigenes encoding enzymes involved in gluconeogenesis (e.g., phosphoenolpyruvate, glycerate- $1,3 \mathrm{P}_{2}$, fructose- $1,6 \mathrm{P}_{2}$, glucose- $1 \mathrm{P}$ ) were up-regulated, while the majority of unigenes involved in glycolysis (e.g., glycerate-3P, glyceraldehyde3P) were down-regulated (Figs. 4, 5). Based on these results, it can be hypothesized that more glucose-1P might be partitioned into the starch and sucrose metabolic pathways for starch biosynthesis in response to nitrogen starvation. 


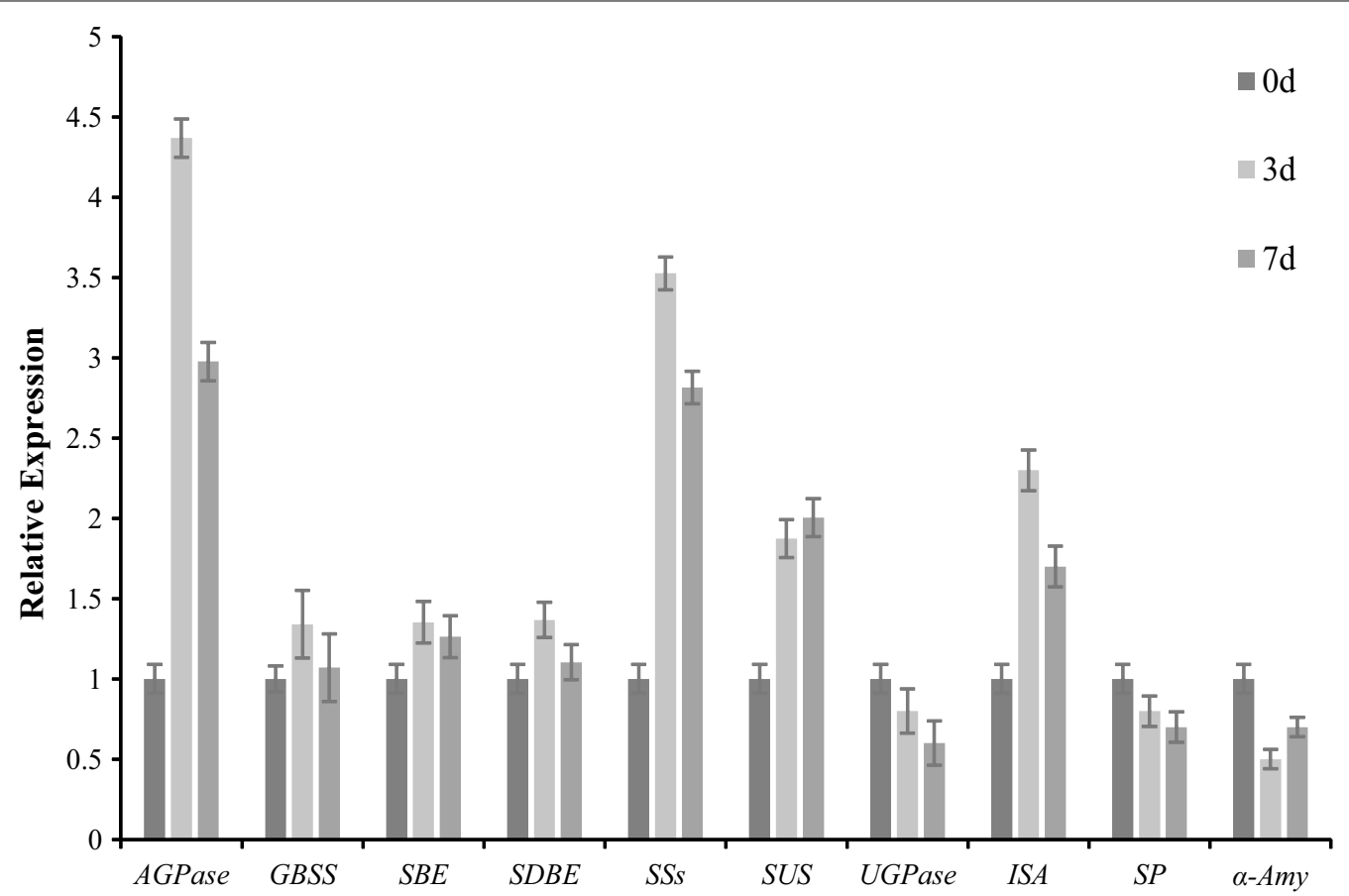

Fig. 4 Validation of the expression of candidate genes during nitrogen starvation by quantitative Real-time RT-PCR analysis. The expression at 0 day was set as 1, and the relative expression level was calculated for several genes. AGPase ADP-glucose pyrophosphorylase, GBSS granule bound starch synthase, SBE starch-branching enzyme, SDBE starch-debranching enzyme, SSs soluble starch synthase, SUS sucrose synthase, UGPase UDP-glucose pyrophosphorylase, ISA isoamylase, SP sucrose phosphorylase

Glucose-1P can be converted into UDP-glucose and GDP-glucose, which potentially compete with starch biosynthesis for substrates. UDP-glucose is generated from glucose-1P through UTP-glucose-1-phosphate uridylyltransferase and is further catalyzed by UDP-glucose 6-dehydrogenase to produce UDP-alpha-D-glucuronate [31]. Transcripts encoding UDP-glucose 6-dehydrogenase were found to be significantly down-regulated under nitrogen starvation (Fig. 5). In contrast, no significant changes in expression were observed for transcripts encoding enzymes in the GDP-glucose branch of the pathway. Overall, under nitrogen starvation, genes involved in starch and related pathways had been activated and led to starch accumulation.

\section{Pathways repressed by nitrogen starvation}

We observed that a substantial number of DEGs were down-regulated under nitrogen starvation and led to significant changes in some metabolic pathways, including nitrogen metabolism, photosynthesis, amino acid biosynthesis, unsaturated fatty acid biosynthesis, and fatty acid degradation (Fig. 3). It is noteworthy that 30 DEGs were found to be significantly enriched in nitrogen metabolism pathways (Fig. 3). For example, genes encoding nitrate reductase (e.g., nas B) and ferredoxin-nitrite reductase (e.g., nir A) were down-regulated during nitrogen starvation, which indicated that the nitrite and $\mathrm{NH}_{3}$ contents were decreased. In addition, the expression of genes encoding glutamine synthetase (e.g., gln A) and glutamate synthase (e.g., GLT 1) were also down-regulated, which suggested that the L-glutamine (L-Gln) and L-glutamate (L-Glu) contents were also decreased under nitrogen starvation (Fig. 5).

DEGs associated with the photosynthetic pathways were significantly down-regulated during nitrogen starvation (Fig. 5). For example, expression of unigenes involved in photosystems II (e.g., Psb A, Psb E, Psb O, and Psb U) and photosynthetic electron transport (e.g., Pet E, Pet F, and Pet $\mathrm{H}$ ) were significantly down-regulated at 3 and 7 days under nitrogen starvation compared to the 0 day control. To determine whether the level of chlorophyll content was decreased under nitrogen starvation, the contents of chlorophyll $a$ and chlorophyll $b$ were measured. The results indicated that the contents of chlorophyll $a$ and chlorophyll $b$ gradually decreased after nitrogen starvation stress was induced (Additional file 9: Figure S7). These results indicated that a progressive loss of photosynthetic pigments accompanied the decreased expression of genes in the photosynthetic pathways in duckweed during nitrogen starvation stress. 


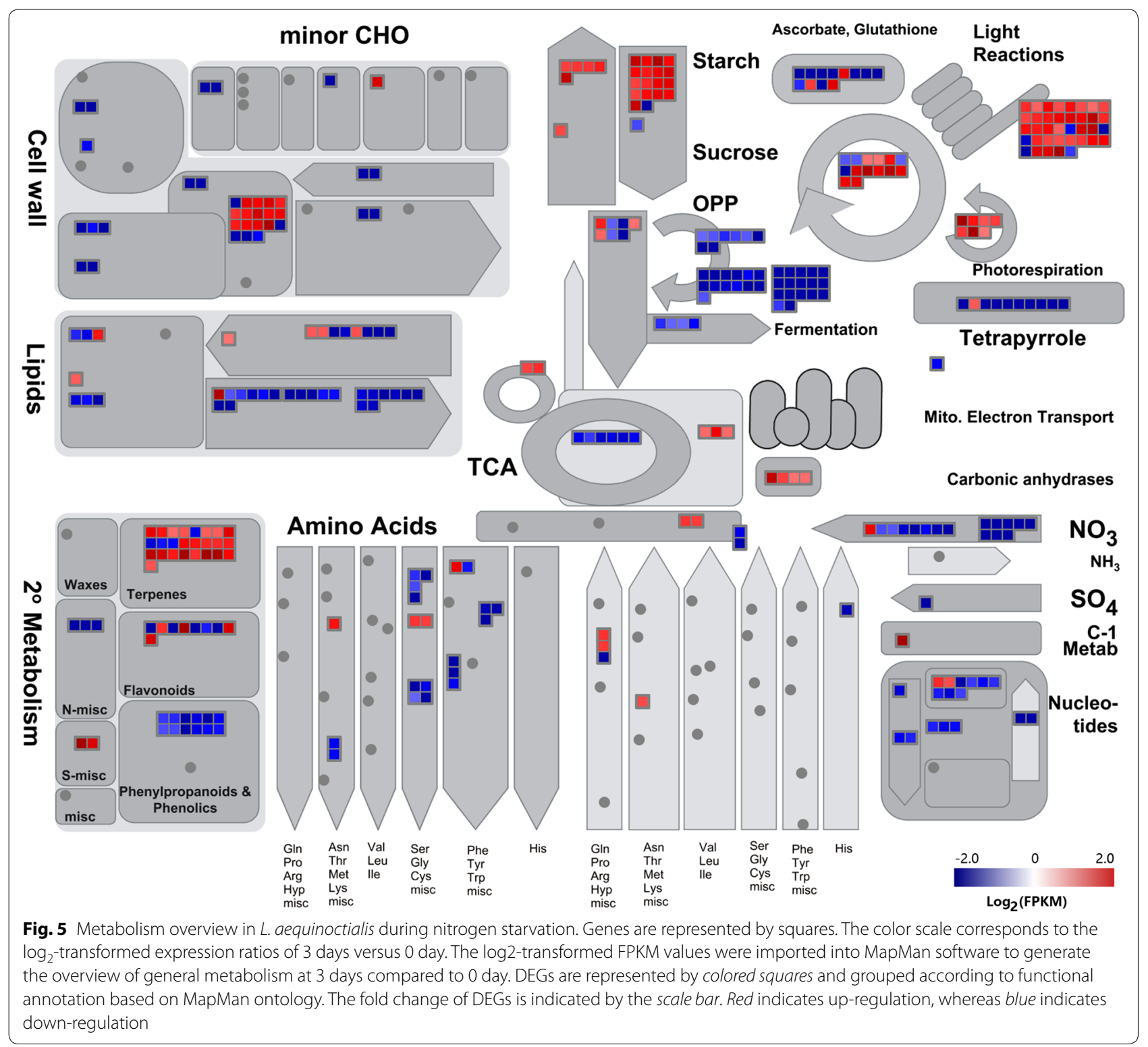

Genes encoding enzymes involved in fatty acid-related pathways were down-regulated, including genes involved in the biosynthesis of unsaturated fatty acid, alpha-linolenic acid metabolism, and fatty acid degradation (Fig. 3). These genes were down-regulated and may then lead to a decrease in the fatty acid biosynthesis.

Under nitrogen starvation, the transcript levels of genes involved in amino acid metabolism were also affected. Generally, the contents of protein were decreased, and the expression of genes involved in amino acid biosynthesis were down-regulated under nitrogen starvation. Consistent with the lower expression of transcripts, the protein content was decreased and might activate starch accumulation under nitrogen starvation (Figs. 3, 5).

\section{Metabolite analysis validates the effect of transcriptome} changes on carbon participation

The effects of the transcriptome changes discussed in the previous sections were validated using liquid chromatography-tandem mass spectrometry (LC-MS/ MS) analysis of specific metabolites in L. aequinoctialis 6000 during nitrogen starvation. The results showed that more ADP-Glc accumulated at 3 and 7 days under nitrogen starvation compared to the 0 day control (Fig. 6). In contrast, lower levels of UDP-Glc accumulated at 3 and 7 days under nitrogen starvation compared to the samples at 0 day (Fig. 6). Furthermore, the enzyme activities of AGPase and UGPase under nitrogen starvation were determined, and the results 


\begin{tabular}{|c|ccc|}
\hline & & & \\
& $0 \mathrm{~d}$ & $3 \mathrm{~d}$ & $7 \mathrm{~d}$ \\
\hline ADP-Glc & $5.6 \pm 1.0$ & $30.5 \pm 0.9$ & $55.3 \pm 2.9$ \\
UDP-Glc & $168 \pm 7.5$ & $96 \pm 8.9$ & $34 \pm 5.0$ \\
Glc-1P & $55 \pm 2.7$ & $70 \pm 3.8$ & $75 \pm 3.9$ \\
\hline Glc-6P & $172 \pm 12.1$ & $255 \pm 21.0$ & $285 \pm 26.2$ \\
\hline Fru-6P & $52 \pm 2.1$ & $74 \pm 3.3$ & $83 \pm 3.9$ \\
\hline Glycerol-3P & $11.2 \pm 1.0$ & $9.5 \pm 0.8$ & $8 \pm 0.7$ \\
Pyruvate & $109 \pm 10.2$ & $92 \pm 7.0$ & $75 \pm 6.3$ \\
\hline PEP & $30 \pm 5.0$ & $36 \pm 6.4$ & $40 \pm 5.8$ \\
\hline
\end{tabular}

Fig. 6 Heatmap of metabolite contents of duckweed fronds during nitrogen starvation. Fronds were collected at different time points and used for metabolite content measurement. The fold change of contents is indicated by the scale bar. Red indicates a higher content, while yellow indicates a lower content

showed that the activity of AGPase was significantly increased, while the activity of UGPase dramatically decreased under nitrogen starvation, which was consistent with the transcriptome and metabolism results (Figs. 6, 7). In addition, the contents of $\alpha$-ketoglutarate and oxaloacetate, which are products of the TCA cycle, also increased during nitrogen starvation. It is notable that the contents of citrate and isocitrate also decreased during nitrogen starvation.

We also measured the metabolites of glycolysis and gluconeogenesis during nitrogen starvation. The results showed that fructose-1,6P and fructose-6P significantly increased during nitrogen starvation (Fig. 6). Thus, it

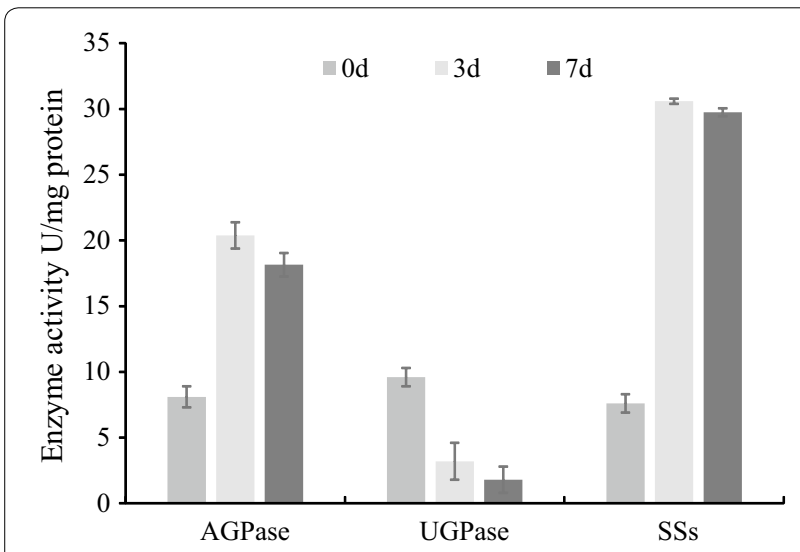

Fig. 7 The enzyme activity of AGPase, UGPase, and SSs during nitrogen starvation at 0,3 , and 7 days. Fronds were collected at different time points and used for a starch metabolism-related enzymatic activity assay after nitrogen starvation. All data are presented as the mean of triplicate measurements \pm standard deviation can be assumed that $\alpha$-ketoglutarate and oxaloacetate generated from the TCA cycle will more likely affect starch synthesis through gluconeogenesis. In addition, the levels of glucose-6P and glucose-1P were significantly decreased at 3 and 7 days under nitrogen starvation conditions compared to the 0 day control (Fig. 6). These changes in metabolite levels during nitrogen starvation are approximately consistent with the gene expression changes in the transcriptome (Figs. 3, 5). Therefore, the mRNA expression patterns induced by nitrogen starvation have an effect on the operation of related metabolic pathways. Based on these results, it can be concluded that the increase in ADP-glucose and starch contents under nitrogen starvation are a consequence of increased output from gluconeogenesis and TCA pathways, which are accompanied by the reduction in lipids and pectin biosynthesis.

\section{Discussion}

\section{Overview of transcriptome dynamics of Lemna aequinoctialis during nitrogen starvation}

Previous studies have shown that starch accumulation in duckweed can be induced by manipulating growth conditions, such as nutrient levels, temperature, $\mathrm{pH}$, light intensity, and photoperiod [4]. Nutrient starvation, especially nitrogen starvation, is the most typical way to induce starch biosynthesis in duckweed [4]. Multiple changes occurred in transcripts involved with nitrogen, carbon, and energy metabolism pathways during nitrogen starvation. These changes included genes encoding proteins involved in the transfer of ammonium groups and in nitrogen assimilation and transport. In addition, these changes are also accompanied by increases in many genes associated with oxidative phosphorylation, which reflects the attempt by cells to compensate for energy deficiency during nitrogen starvation. In contrast, the transcript abundance of many genes involved in photosynthesis and protein biosynthesis was significantly decreased. In addition, the transcriptome and metabolite analysis indicated that carbon metabolism shifted from glucose synthesis to utilization and storage of starches rather than fatty acids (Fig. 5). These data defined the transcript profiles that reflect the global response to nitrogen limitation over a 7-day period in duckweed. The increased starch synthesis and storage might suggest that duckweed can serve as an important source of bioethanol feedstock, and can be utilized as a model system to study the underlying mechanisms of starch biosynthesis.

However, it is noteworthy that the higher starch accumulation under nitrogen starvation in duckweed is attained at the expense of retarded growth resulting from significant reductions in photosynthesis and fatty acid biosynthesis. 


\section{How was starch accumulated in L. aequinoctialis during nitrogen starvation?}

Starch is one of the major storage compounds of duckweed and usually accumulates under stressed conditions (e.g., nitrogen starvation), which is an adaptive strategy gained during the long evolutionary process to enable the plant to survive the adverse environment [32]. The starch accumulation was significantly induced in L. aequinoctialis under nitrogen starvation, from an initial level around $20 \%$ of dry weight to a maximum around $60 \%$ (Fig. 1A). Strikingly, it should be noted that the starch content was continuously accumulated during nitrogen starvation for up to 9 days in L. aequinoctialis (Fig. 1A). Similarly, a large amount of starch is usually accumulated during nitrogen starvation stress in algae. It has been shown that an increase of $41 \%$ in starch content was observed after $24 \mathrm{~h}$ nitrogen starvation in $C$. reinhardtii, and the starch content was almost doubled that of the control after $144 \mathrm{~h}$ treatment under nitrogen starvation [34]. However, algae usually first stores carbon sources as starches and then quickly converts the starches to triacylglycerol (TAG) in response to nitrogen starvation stress [33]. It has been reported that the total fatty acid levels increased by about $50 \%$ after $24 \mathrm{~h}$ of nitrogen starvation, and increased further to 2- and 3.8 -fold after 48 and $144 \mathrm{~h}$ in C. reinhardtii [34]. In contrast, the interconversion of starch to lipids dose not seemingly occur in duckweed. Our transcriptome data are in coincidence with this notion because the expression of massive genes involved in fatty acid synthesis were significantly down-regulated during nitrogen starvation. One of the possible reasons for this down-regulation might be due to the fact that acetyl-CoA is the substrate for both fatty acid synthesis and glycolysis/ gluconeogenesis. It is noteworthy that even though the total fatty acid level was decreased under nitrogen starvation, the TAG content might not change significantly because TAG only accounts for $1 \%$ (dry weight) of duckweed biomass [35]. As duckweed is capable of substantial starch accumulation compared to the other plants, it might be in great need to reduce fatty acid biosynthesis and move more substrates toward starch synthesis. This hypothesis is supported by the significant up-regulation of genes involved in glycolysis/gluconeogenesis, which might lead more acetyl-CoA to convert to glucose-1P. In this respect, total fatty acid content was decreased, and more substrates (e.g., acetyl-CoA) were then dedicated to starch synthesis under nitrogen starvation. This dedication may partially explain why duckweed has a higher capacity for starch accumulation compared to other plants. Moreover, the shift of carbon flux from amino acids to starch under nitrogen starvation in duckweed further reinforced that notion that there exists ultimate link between carbon and nitrogen metabolisms in plants.

Although several previous studies have revealed that starch accumulation is a general response to nutrient deficiency in some plants, the mechanisms underlying starch accumulation under nutrient deficiency still remain elusive. As preliminary analysis of starch biosynthesis under nitrogen starvation has already been conducted in previous transcriptome and physiological studies [36-38], we carried out a more comprehensive analysis of the gene network during nitrogen starvation in this study by taking advantage of the integrated transcriptome and metabolism analysis. The results showed that multiple changes occurred in the expression of genes involved in nitrogen, carbon, and energy metabolism during nitrogen starvation conditions. The expression of genes associated with photosynthetic machinery was significantly reduced, which was accompanied by significant decreases in the contents of chlorophyll $a$ and chlorophyll $b$ (Additional file 9: Figure S7). Additionally, the expression of many genes involved in protein synthesis was reduced under nitrogen starvation conditions, which indicated that protein synthesis was likely inhibited during nitrogen starvation (Fig. 5).

The bulk of the ammonium in plant cells targets the chloroplast, where almost all the enzymes necessary for the incorporation of ammonia into carbon skeletons through the GS/GOGAT pathway are located. The glutamine synthases are involved in the catalysis of glutamine formation from ammonia and glutamate. Glutamate is among one of the few key molecules that links the amino acid metabolic pathways with the carbohydrate and lipid metabolic pathways [39]. Our results indicated that DEGs encoding proteins related to Glu and Gln biosynthesis were dramatically down-regulated under nitrogen stress, which led to an influx of metabolites into carbohydrate and lipid biosynthetic pathways (Additional file 10: Figure S8).

The flux of carbon metabolism is mainly derived from the synthesis of glucose and ends in utilization and storage as starch. To obtain more starch accumulation, two types of strategies will usually be adopted: more substrates from other metabolic pathways being dedicated to starch and sucrose metabolism or a lower starch degradation rate. Metabolic profiling can provide information on the dynamic metabolic status of a living system during stress (e.g., nitrogen starvation). Thus the metabolic profiles might help to systematically decipher the affected pathways and underlying mechanisms after stress treatment and help to corroborate the transcriptome data. Previous studies on metabolism flux have dramatically contributed towards improving our understanding of 
resource allocation and regulation in model plant Arabidopsis [32].

UDP-glucose plays an important role in the allocation of carbon metabolism, and it has four branches to the next metabolite, sucrose, glycogen, UDP-glucuronate, and glucose-1P. Thus, UDP-glucose is one of the important substrates for starch synthesis. In this study, DEGs involved in the UDP-glucuronate and pectin branches were significantly down-regulated during nitrogen starvation, which directed more substrates to the starch synthesis pathway (Fig. 8). Moreover, DEGs encoding the key enzymes in starch synthesis (e.g., AGPs and SSs) also had high expression levels and then catalyzed all the metabolic substrates to be efficiently transformed into starch. The transcriptome data and metabolic analysis defined the global transcript profiling and metabolic changes in response to nitrogen starvation in duckweed. The results indicated that duckweed reduced the branch to pectin biosynthesis and integrated all metabolite substrates into starch synthesis, which ultimately contributed to the higher starch accumulation during nitrogen starvation.

Taking all of the transcriptomic and metabolic changes into account, we concluded that nitrogen deprivation caused the suppression of global metabolism status and redirected the flux from the amino acid and cell wall (especially pectin) biosynthesis toward the biosynthesis of starch.

\section{Conclusions}

Starch metabolism is a complex process triggered by changes in the levels of numerous transcripts and metabolites. Using next-generation sequencing, the

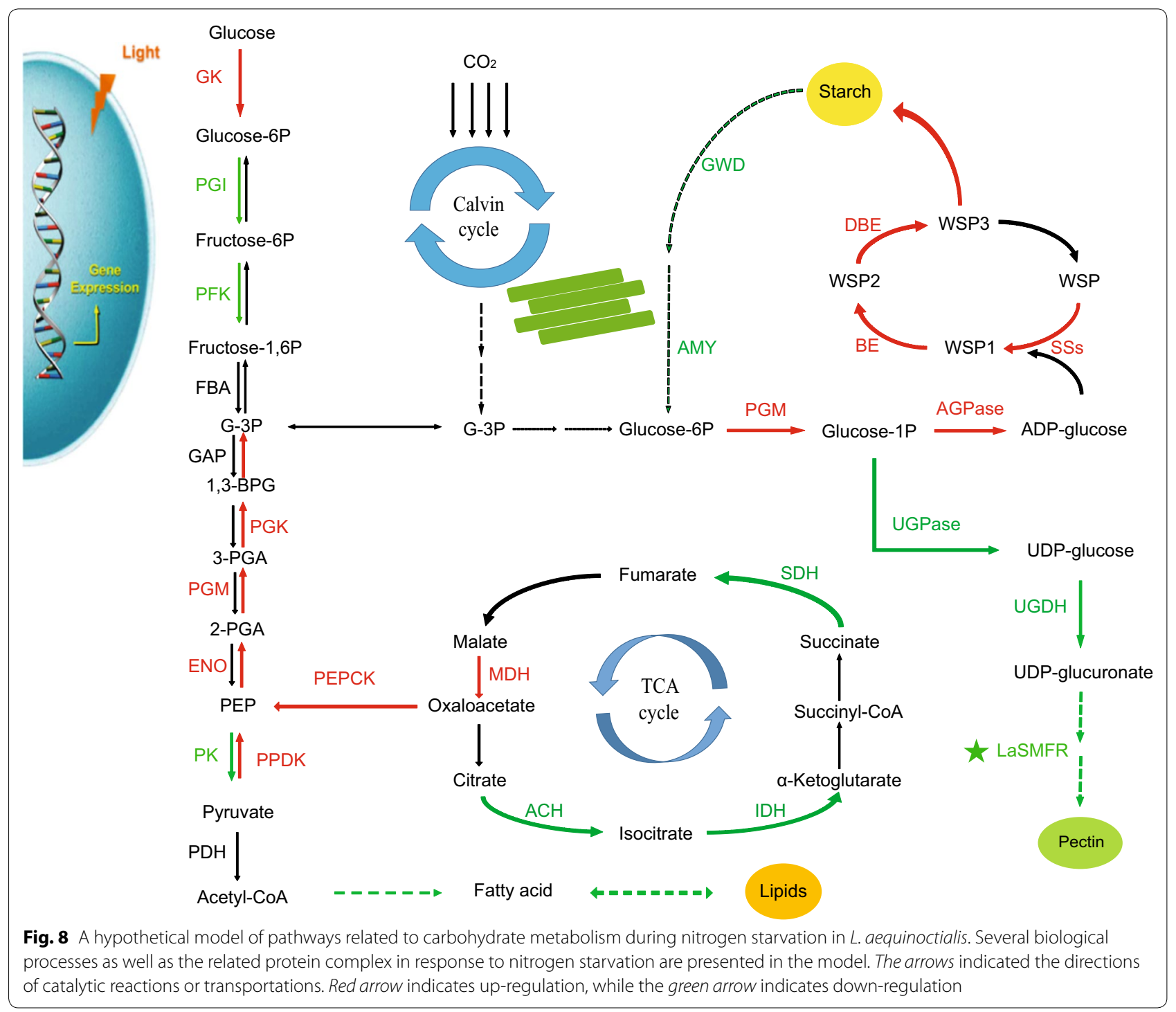


transcriptome of Lemna aequinoctialis was analyzed during nitrogen starvation. The genes involved in nitrogen metabolism, protein and amino acid metabolism, starch and sucrose metabolism, and lipid metabolism were evaluated. The transcriptome results revealed insights into the putative molecular mechanisms underlying starch accumulation in duckweed during nitrogen starvation. Moreover, the results of metabolite analysis corroborated the transcriptome data. Overall, the results presented here describe the dynamic transcriptome changes in $L$. aequinoctialis and reveal the complex mechanisms in starch accumulation. The genes identified as being involved in starch metabolism represent excellent candidates for further genetic improvement of starch production in duckweed.

\section{Additional files}

Additional file 1: Table S1. Assembly quality statistics of $L$. aequinoctialis 6000 .

Additional file 2: Table S2. List of the whole duckweed unigenes with annotations expressed in three stages under nitrogen starvation.

Additional file 3: Figure S1. Statistics of the annotation of unigenes in public databases.

Additional file 4: Figure S2. Classification of Gene Ontology of assembled unigenes.

Additional file 5: Figure S3. Histogram presentation of clusters of orthologous groups (COGs) classification of unigenes.

Additional file 6: Figure S4. Pathway assignment based on the KEGG database.

Additional file 7: Figure S5. GO enrichment analysis of differentially expressed genes in L. aequinoctialis during nitrogen starvation.

Additional file 8: Figure S6. KEGG enrichment analysis of differentially expressed genes in L. aequinoctialis during nitrogen starvation.

Additional file 9: Figure S7. Decreased chlorophyll content of L. aequinoctialis 6000 under nitrogen starvation. The contents of chlorophyll a and chlorophyll b (mg/g) were measured in a spectrophotometric assay under nitrogen starvation conditions at 0, 3 and 7 days.

Additional file 10: Figure S8. Validation of the expression of candidate genes involved in glycolysis and gluconeogenesis under nitrogen starvation by quantitative Real-time PCR analysis.

\section{Abbreviations}

ABA: abscisic acid; AGP: ADP-glucose pyrophosphorylase; GBSS: granule bound starch synthase; SBE: starch-branching enzyme; DEGs: differential expression genes; TAG: triacylglycerol; PAS: periodic Acid-Schiff; SH: Schenk and Hildebrandt; SSs: soluble starch synthase; DW: dry weight; FPKM: fragments per kilobase of transcripts per million mapped fragments; KEGG: Kyoto Encyclopedia of Genes and Genomes; NGS: next-generation sequencing; PE: paired-end; FW: fresh weight; log2FC: log2 fold change; GS: glutamine synthase.

\section{Authors' contributions}

CJY, RBH, and GKZ designed the project and defined the overall experimental approach. CJY, YW, and SMW conducted all cultivation and sampling for the transcriptome sequencing. CJY, ZTB, and QL conducted the transcriptome analysis. XWZ, YBM, and GQ conducted all enzyme activity analysis and metabolomic measurements and associated data analyses. All authors read and approved the final manuscript.

\begin{abstract}
Author details
${ }^{1}$ Shandong Provincial Key Laboratory of Energy Genetics, Qingdao Engineering Research Center of Biomass Resources and Environment, Qingdao Institute of Bioenergy and Bioprocess Technology, Chinese Academy of Sciences, Qingdao 266101, People's Republic of China. ${ }^{2}$ University of Chinese Academy of Sciences, Beijing 100049, People's Republic of China. ${ }^{3}$ College of Life Sciences, China Agricultural University, Beijing 100094, People's Republic of China.

${ }^{4}$ Guangzhou Genedenovo Biotechnology Co., Ltd, Guangzhou 510006, China.
\end{abstract}

\section{Acknowledgements}

The authors would like to acknowledge Guangzhou Genedenovo Biotechnology Co., Ltd for help with transcriptome analysis.

\section{Competing interests}

The authors declare that they have no competing interests.

\section{Availability of supporting data}

All supplemental data have been made freely available; specific data repository information are specified within the manuscript. The data of RNA sequencing were deposited at Sequence Read Archive (SAMN06266583) with accession no. SRR5660714 in National Center for Biotechnology Information (NCBI).

\section{Funding}

This study was funded by the National Key Technology Research and Development Program of China (2015BAD15B01), National Natural Science Foundation of China (No. 31600285,No.U1632140), major science and technology projects of Shandong province (2015ZDJS03002, 2016ZDJS10A04, 2016GNC113020), and the Taishan Scholar Program of Shandong.

\section{Publisher's Note}

Springer Nature remains neutral with regard to jurisdictional claims in published maps and institutional affiliations.

Received: 16 February 2017 Accepted: 16 June 2017

Published online: 26 June 2017

\section{References}

1. Qiu HG, Huang JK, Keyzer M, van Veen W, Rozelle S, Fisher G, et al. Biofuel development, food security and the use of Marginal land in China. J Environ Qual. 2011;40(4):1058-67.

2. Ragauskas AJ, Williams CK, Davison BH, Britovsek G, Cairney J, Eckert $C A$, et al. The path forward for biofuels and biomaterials. Science. 2006:311(5760):484-9.

3. Sun Y, Cheng JY. Hydrolysis of lignocellulosic materials for ethanol production: a review. Biores Technol. 2002;83(1):1-11.

4. Cui W, Cheng JJ. Growing duckweed for biofuel production: a review. Plant Biol (Stuttg). 2015;17:16-23.

5. Bog M, Baumbach H, Schween U, Hellwig F, Landolt E, Appenroth KJ. Genetic structure of the genus Lemna L. (Lemnaceae) as revealed by amplified fragment length polymorphism. Planta. 2010;232(3):609-19.

6. Xu JL, Cui WH, Cheng JJ, Stomp AM. Production of high-starch duckweed and its conversion to bioethanol. Biosyst Eng. 2011;110(2):67-72.

7. Xiao Y, Fang $Y$, Jin $Y L$, Zhang GH, Zhao H. Culturing duckweed in the field for starch accumulation. Ind Crop Prod. 2013;48:183-90.

8. Cheng JJ, Stomp AM. Growing duckweed to recover nutrients from wastewaters and for production of fuel ethanol and animal feed. Clean Soil Air Water. 2009;37(1):17-26.

9. Reid MS, Bieleski RL. Response of Spirodela oligorrhiza to phosphorus deficiency. Plant Physiol. 1970;46(4):609-13.

10. Sree KS, Adelmann K, Garcia C, Lam E, Appenroth KJ. Natural variance in salt tolerance and induction of starch accumulation in duckweeds. Planta. 2015;241:1395-404.

11. Wang W, Kerstetter RA, Michael TP. Evolution of genome size in duckweeds (Lemnaceae). J Bot. 2011;2011:1-9.

12. Upadhyay S, Phukan UJ, Mishra S, Shukla RK. De novo leaf and root transcriptome analysis identified novel genes involved in steroidal sapogenin biosynthesis in Asparagus racemosus. BMC Genom. 2014;15:746. 
13. Zhang M, Kong $X$, Xu X, Li C, Tian H, Ding Z. Comparative transcriptome profiling of the maize primary, crown and seminal root in response to salinity stress. PLoS ONE. 2015;10(3):e0121222.

14. Tao $X$, Fang $Y$, Xiao $Y$, Jin $Y L, M a X R$, Zhao $Y$, et al. Comparative transcriptome analysis to investigate the high starch accumulation of duckweed (Landoltia punctata) under nutrient starvation. Biotechnol Biofuels. 2013;6(1):72.

15. Wang WQ, Wu YR, Messing J. RNA-Seq transcriptome analysis of Spirodela dormancy without reproduction. Bmc Genomics. 2014;15:60.

16. Van Hoeck A, Horemans N, Nauts R, Van Hees M, Vandenhove H, Blust R. Lemna minor plants chronically exposed to ionising radiation: RNA-seq analysis indicates a dose rate dependent shift from acclimation to survival strategies. Plant Sci. 2017;257:84-95.

17. Yu C, Sun C, Yu L, Zhu M, Xu H, Zhao J, et al. Comparative analysis of duckweed cultivation with sewage water and $\mathrm{SH}$ media for production of fuel ethanol. PLoS ONE. 2014;9(12):e115023.

18. Wang W, Messing J. Analysis of ADP-glucose pyrophosphorylase expression during turion formation induced by abscisic acid in Spirodela polyrhiza (greater duckweed). BMC Plant Biol. 2012;12:5.

19. Grabherr MG, Haas BJ, Yassour M, Levin JZ, Thompson DA, Amit I, et al. Full-length transcriptome assembly from RNA-Seq data without a reference genome. Nat Biotechnol. 2011;29(7):644-52.

20. Pertea G, Huang X, Liang F, Antonescu V, Sultana R, Karamycheva S, et al. TIGR gene indices clustering tools (TGICL): a software system for fast clustering of large EST datasets. Bioinformatics. 2003;19(5):651-2.

21. Li B, Dewey CN. RSEM: accurate transcript quantification from RNASeq data with or without a reference genome. BMC Bioinformatics. 2011;12:323.

22. Anders $\mathrm{S}$, Huber W. Differential expression analysis for sequence count data. Genome Biol. 2010;11(10):R106.

23. Young MD, Wakefield MJ, Smyth GK, Oshlack A. Gene ontology analysis for RNA-seq: accounting for selection bias. Genome Biol. 2010;11 (2):R14.

24. Xie C, Mao X, Huang J, Ding Y, Wu J, Dong S, et al. KOBAS 2.0: a web server for annotation and identification of enriched pathways and diseases. Nucleic Acids Res. 2011;39:W316-22.

25. Schmittgen TD, Livak KJ. Analyzing real-time PCR data by the comparative C(T) method. Nat Protoc. 2008;3(6):1101-8.

26. Crumpton-Taylor M, Pike M, Lu KJ, Hylton CM, Feil R, Eicke S, et al. Starch synthase 4 is essential for coordination of starch granule formation with chloroplast division during Arabidopsis leaf expansion. New Phytol. 2013;200(4):1064-75.

27. Tarrago JF, Nicolas G. Starch degradation in the cotyledons of germinating lentils. Plant Physiol. 1976;58(5):618-21.

28. Nakamura H, Yuasa I, Umetsu K, Nakagawa M, Nanba E, Kimura K. The rearrangement of the human alpha(1)-acid glycoprotein/orosomucoid gene: evidence for tandemly triplicated genes consisting of two AGP1 and one AGP2. Biochem Biophys Res Commun. 2000;276(2):779-84.
29. Jenner CF, Denyer K, Hawker JS. Caution on the use of the generally accepted methanol precipitation technique for the assay of soluble starch synthase in crude extracts of plant-tissues. Aust J Plant Physiol. 1994;21(1):17-22.

30. Hedhly A, Vogler H, Schmid MW, Pazmino D, Gagliardini V, Santelia D, et al. Starch turnover and metabolism during flower and early embryo development. Plant Physiol. 2016;172(4):2388-402.

31. Li M, Chen T, Gao T, Miao Z, Jiang A, Shi L, et al. UDP-glucose pyrophosphorylase influences polysaccharide synthesis, cell wall components, and hyphal branching in Ganoderma lucidum via regulation of the balance between glucose-1-phosphate and UDP-glucose. Fungal Genet Biol. 2015;82:251-63.

32. Smith AM. Starch in the Arabidopsis plant. Starch-Starke. 2012;64(6):421-34.

33. Siaut M, Cuine S, Cagnon C, Fessler B, Nguyen M, Carrier P, et al. Oil accumulation in the model green alga Chlamydomonas reinhardtii: characterization, variability between common laboratory strains and relationship with starch reserves. BMC Biotechnol. 2011;11:7.

34. Wase N, Black PN, Stanley BA, DiRusso CC. Integrated quantitative analysis of nitrogen stress response in Chlamydomonas reinhardtii using metabolite and protein profiling. J Proteome Res. 2014;13(3):1373-96.

35. Yan Y, Candreva J, Shi H, Ernst E, Martienssen R, Schwender J, et al. Survey of the total fatty acid and triacylglycerol composition and content of 30 duckweed species and cloning of a Delta6-desaturase responsible for the production of gamma-linolenic and stearidonic acids in Lemna gibba. BMC Plant Biol. 2013;13:201

36. Liu Y, Fang Y, Huang M, Jin Y, Sun J, Tao X, et al. Uniconazole-induced starch accumulation in the bioenergy crop duckweed (Landoltia punctata) II: transcriptome alterations of pathways involved in carbohydrate metabolism and endogenous hormone crosstalk. Biotechnol Biofuels. 2015;8:64.

37. Liu Y, Fang Y, Huang M, Jin Y, Sun J, Tao X, et al. Uniconazole-induced starch accumulation in the bioenergy crop duckweed (Landoltia punctata) I: transcriptome analysis of the effects of uniconazole on chlorophyll and endogenous hormone biosynthesis. Biotechnol Biofuels. 2015;8:57.

38. Tao X, Fang Y, Xiao Y, Jin YL, Ma XR, Zhao Y, et al. Comparative transcriptome analysis to investigate the high starch accumulation of duckweed (Landoltia punctata) under nutrient starvation. Biotechnol Biofuels. 2013;6:72.

39. Hirai MY, Yano M, Goodenowe DB, Kanaya S, Kimura T, Awazuhara M, et al. Integration of transcriptomics and metabolomics for understanding of global responses to nutritional stresses in Arabidopsis thaliana. Proc Natl Acad Sci USA. 2004;101(27):10205-10.

\section{Submit your next manuscript to BioMed Central and we will help you at every step:}

- We accept pre-submission inquiries

- Our selector tool helps you to find the most relevant journal

- We provide round the clock customer support

- Convenient online submission

- Thorough peer review

- Inclusion in PubMed and all major indexing services

- Maximum visibility for your research

Submit your manuscript at www.biomedcentral.com/submit
() BioMed Central 